\title{
Mapping linked quantitative trait loci via residual maximum likelihood
}

\author{
FE Grignola, Q Zhang, I Hoeschele* \\ Department of Dairy Science, Virginia Polytechnic Institute and State University, \\ Blacksburg, VA 24061-0315, USA
}

(Received 2 September 1996; accepted 19 August 1997)

\begin{abstract}
Summary - A residual maximum likelihood method is presented for estimation of the positions and variance contributions of two linked QTLs. The method also provides tests for zero versus one QTL linked to a group of markers and for one versus two QTLs linked. A deterministic, derivative-free algorithm is employed. The variance--covariance matrix of the allelic effects at each QTL and its inverse is computed conditional on incomplete information from multiple linked markers. Covariances between effects at different QTLs and between QTLs and polygenic effects are assumed to be zero. A simulation study was performed to investigate parameter estimation and likelihood ratio tests. The design was a granddaughter design with 2000 sons, 20 sires of sons and nine ancestors of sires. Data were simulated under a normal-effects and a biallelic model for variation at each QTL. Genotypes at five or nine equally spaced markers were generated for all sons and their ancestors. Two linked QTLs accounted jointly for 50 or $25 \%$ of the additive genetic variance, and distance between QTLs varied from 20 to $40 \mathrm{cM}$. Power of detecting a second QTL exceeded 0.5 all the time for the 50\% QTLs and when the distance was (at least $30 \mathrm{cM}$ for the $25 \%$ QTLs. An intersection-union test is preferred over a likelihood ratio test, which was found to be rather conservative. Parameters were estimated quite accurately except for a slight overestimation of the distance between two close QTLs.
\end{abstract}

quantitative trait loci / multipoint mapping / residual maximum likelihood / outcross population

Résumé - Détection de gènes liés à effets quantitatifs (QTL) grâce au maximum de vraisemblance résiduelle. On présente une méthode de maximum de vraisemblance résiduelle pour estimer les positions et les contributions à la variabilité génétique de deux QTLs liés. La méthode fournit également des tests de l'existence d'un seul QTL lié à un groupe de marqueurs (par rapport à zéro) ou de deux QTLs (par rapport à un seul). Un algorithme déterministe sans calcul de dérivées est utilisé. La matrice de variancecovariance des effets alléliques à chaque QTL et son inverse est calculée conditionnellement à l'information incomplète sur les marqueurs multiples liés. Les covariances entre les effets aux différents QTLs et entre les effets aux QTLs et les effets polygéniques sont

\footnotetext{
${ }^{*}$ Correspondence and reprints
} 
supposées nulles. Une étude de simulation a été effectuée pour analyser les paramètres estimés et les tests de rapports de vraisemblance. Le schéma expérimental a été un schéma "petites-filles» avec 2000 fils, 20 pères des fils et 9 ancêtres de ces pères. Des données ont été simulées avec un modèle de variation au QTL de type Gaussien ou biallélique. Les génotypes pour cinq ou neuf marqueurs également espacés ont été générés pour tous les fils et leurs anchêtres. Deux QTLs liés expliquaient conjointement $50 \%$ ou $25 \%$ de la variance génétique additive et la distance entre les QTLs variait de $20 \mathrm{CM}$ à $40 \mathrm{CM}$. La puissance de détection d'un second QTL a dépassé 0,5, dans tous les cas pour la situation $50 \%$, et quand la distance entre QTLs était supérieure ou égale à 30 CM pour la situation $25 \%$. Le test d'un QTL par rapport à deux QTLs correspond à la réunion de deux tests. On l'a trouvé plutôt conservatif. Les paramètres ont été estimés avec une grande précision excepté la distance entre deux QTLs proches qui a été légèrement surestimée.

locus de caractère quantitatif / cartographie multipoint / maximum de vraisemblance résiduelle / population consanguine

\section{INTRODUCTION}

A variety of methods for the statistical mapping of quantitative trait loci (QTL) exist. While some methods analyze squared phenotypic differences of relative pairs (eg, Haseman and Elston, 1972; Gotz and Ollivier, 1994), most methods analyze the individual phenotypes of pedigree members. Main methods applied to livestock populations are maximum likelihood (ML) (eg, Weller, 1986; Lander and Botstein, 1989; Knott and Haley, 1992), least-squares (LS) as an approximation to ML (eg, Weller et al, 1990; Haley et al, 1994; Zeng, 1994), and a combination of ML and LS referred to as composite interval mapping (Zeng, 1994) or multiple QTL mapping (Jansen, 1993). These methods were developed mainly for line crossing and, hence, cannot fully account for the more complex data structures of outcross populations, such as data on several families with relationships across families, incomplete marker information, unknown number of QTL alleles in the population and varying amounts of data on different QTLs or in different families.

Recently, Thaller and Hoeschele (1996a, b) and Uimari et al (1996) implemented a Bayesian method for QTL mapping using single markers or all markers on a chromosome, respectively, via Markov chain Monte Carlo algorithms, and applied the analyses to simulated granddaughter designs identical to those in the present study. Hoeschele et al (1997) showed that the Bayesian analysis can accommodate either a biallelic or a normal-effects QTL model. While the Bayesian analysis was able to account for pedigree relationships both at the QTL and for the polygenic component, and gave good parameter estimates, it was very demanding in terms of computing time, in particular when fitting two QTLs (Uimari and Hoeschele, 1997).

Therefore, Grignola et al (1996a) developed a residual maximum likelihood method, using a deterministic, derivative-free algorithm, to map a single QTL. Hoeschele et al (1997) showed that this method can be considered as an approximation to the Bayesian analysis fitting a normal-effects QTL model. In the normaleffects QTL model postulated by the REML analysis, the vector of QTL allelic effects is random with a prior normal distribution. The REML analysis builds on earlier work by Fernando and Grossman (1989), Cantet and Smith (1991) and Goddard (1992) on best linear unbiased prediction of QTL allelic effects by extending 
it to the estimation of QTL, polygenic, and residual variance components and of QTL location, using incomplete information from multiple linked markers.

$\mathrm{Xu}$ and Atchley (1995) performed interval mapping using maximum likelihood based on a mixed model with random QTL effects, but these authors fitted additive genotypic effects rather than allelic effects at the QTL, with variance-covariance matrix proportional to a matrix of proportions of alleles identical-by-descent, and assumed that this matrix was known. Their analysis was applied to unrelated fullsib pairs. In order to account for several QTLs on the same chromosome, Xu and Atchley (1995) used the idea behind composite interval mapping and fitted variances at the two markers flanking the marker bracket for a QTL. This approach, however, is not appropriate for multi-generational pedigrees, as effects associated with marker alleles erode across generations owing to recombination. It is also problematic for outbred populations, where incomplete marker information causes the flanking and next-to-flanking markers to differ among families.

In this paper, we extend the REML method of Grignola et al (1996a) to the fitting of multiple linked QTLs. While the extension is general for any number of linked QTLs, we apply the method to simulated granddaughter designs by fitting either one or two QTLs.

\section{METHODOLOGY}

\section{Mixed linear model}

The model is identical to that of Grignola et al (1996a), except that it includes effects at several $(t)$ QTLs, and it can be written as:

$$
\mathbf{y}=\mathbf{X} \boldsymbol{\beta}+\mathbf{Z u}+\mathbf{Z T}\left(\sum_{i=1}^{t} \mathbf{v}_{i}\right)+\mathbf{e}
$$

with

$$
\begin{aligned}
& \operatorname{Var}(\mathbf{v})=\operatorname{Diag}\left\{\operatorname{Var}\left(\mathbf{v}_{i}\right)\right\}, \quad \operatorname{Var}\left(\mathbf{v}_{i}\right)=\mathbf{Q}_{i} \sigma_{v(i)}^{2} \\
& \operatorname{Var}(\mathbf{u})=\mathbf{A} \sigma_{u}^{2}, \quad \operatorname{Var}(\mathbf{e})=\mathbf{R} \sigma_{e}^{2}
\end{aligned}
$$

where $\mathbf{y}$ is a vector of phenotypes, $\mathbf{X}$ is a design-covariate matrix, $\boldsymbol{\beta}$ is a vector of fixed effects, $\mathbf{Z}$ is an incidence matrix relating records to individuals, $\mathbf{u}$ is a vector of residual additive (polygenic) effects, $\mathbf{T}$ is an incidence matrix relating individuals to alleles, $\mathbf{v}_{i}$ is a vector of QTL allelic effects at QTL $i$, $\mathbf{e}$ is a vector of residuals, $\mathbf{A}$ is the additive genetic relationship matrix, $\sigma_{u}^{2}$ is the polygenic variance, $\mathbf{Q}_{i} \sigma_{v(i)}^{2}$ is the variance-covariance matrix of the allelic effects at QTL $i$ conditional on marker information, $\sigma_{v(i)}^{2}$ is the allelic variance at QTL $i$ (or half of the additive variance at QTL $i$ ), $\mathbf{R}$ is a known diagonal matrix, and $\sigma_{e}^{2}$ is residual variance. Each matrix $\mathbf{Q}_{i}$ depends on one unknown parameter, the map position of QTL $i\left(d_{i}\right)$. Parameters related to the marker map (marker positions and allele frequencies) are assumed to be known. The model is parameterized in terms of the unknown parameter's heritability $\left(h^{2}=\sigma_{a}^{2} / \sigma_{p}^{2}\right)$, with $\sigma_{p}^{2}$ being phenotypic and $\sigma_{a}^{2}$ additive genetic variance, fraction of the additive genetic variance explained by the allelic 
effects at QTL $i\left(v_{i}^{2}=\sigma_{v(i)}^{2} / \sigma_{a}^{2} ; i=1, \ldots, t\right)$, the residual variance $\sigma_{e}^{2}$, and QTL map locations $d_{1}, \ldots, d_{i}, \ldots, d_{t}$.

A model equivalent to the animal model in [1] is (Grignola et al, 1996a):

$$
\begin{gathered}
\mathbf{y}=\mathbf{X} \boldsymbol{\beta}+\mathbf{W} \mathbf{u}_{p}+\mathbf{T} \sum_{i=1}^{t} \mathbf{F}_{i} \mathbf{v}_{p}(i) \mathbf{m}+\mathbf{T} \sum_{i=1}^{t} \boldsymbol{\varepsilon}_{i}+\mathbf{e} \\
\operatorname{Var}\left(\mathbf{u}_{p}\right)=\mathbf{A}_{p} \sigma_{u}^{2}, \operatorname{Var}\left(\mathbf{v}_{p(i)}\right)=\mathbf{Q}_{p(i)} \sigma_{v(i)}^{2}, \operatorname{Var}(\mathbf{m})=\Delta_{u} \sigma_{u}^{2} \\
\cdot \operatorname{Var}\left(\boldsymbol{\varepsilon}_{i}\right)=\Delta_{v(i)} \sigma_{v(i)}^{2}, \operatorname{Var}(\mathbf{e})=\mathbf{R} \sigma_{e}^{2}
\end{gathered}
$$

where $\mathbf{W}$ has at most two non-zero elements equal to 0.5 in each row in columns pertaining to the known parents of an individual, $\mathbf{F}_{i}$ is a matrix with up to four non-zero elements per row pertaining to the QTL effects of an individual's parents (Wang et al, 1995; Grignola et al, 1996a), $\mathbf{A}_{p}$ and $\mathbf{Q}_{p(i)}$ are sub-matrices of $\mathbf{A}$ and $\mathbf{Q}$, respectively, pertaining to all animals that are parents, and $\mathbf{m}$ and $\boldsymbol{\varepsilon}_{i}$ are Mendelian sampling terms for polygenic and QTL effects, respectively, with covariance matrices as specified in equation [2]. While $\operatorname{Var}(\mathbf{m})$ is diagonal, $\operatorname{Var}\left(\varepsilon_{i}\right)$ can have some off-diagonal elements in inbred populations (Hoeschele, 1993; Wang et al, 1995).

Note that models [1] and [2] are conditional on a set of QTL map positions (and on marker positions which are assumed to be known). Dependent on the map positions are the matrices $\mathbf{Q}_{i}$ in model [1] and the matrices $\mathbf{F}_{i}$ and $\mathbf{Q}_{p(i)}$ in model [2].

Note furthermore that models [1] and [2] assume zero covariances between effects at different QTLs, and between polygenic and QTL effects. However, selection tends to introduce negative covariances between QTLs (Bulmer, 1985).

A reduced animal model (RAM) can be obtained from model [2] by combining $\mathbf{m}$, the $\boldsymbol{\varepsilon}_{i}(i=1, \ldots, t)$ and $\mathbf{e}$ into the residual. Mixed model equations (MME) can be formed directly for the RAM, or by setting up the MME for model [2] and absorbing the equations in $\mathbf{m}$ and the $\boldsymbol{\varepsilon}_{i}(i=1, \ldots, t)$. The resulting MMEs for the RAM and for $t=2$ QTLs are:

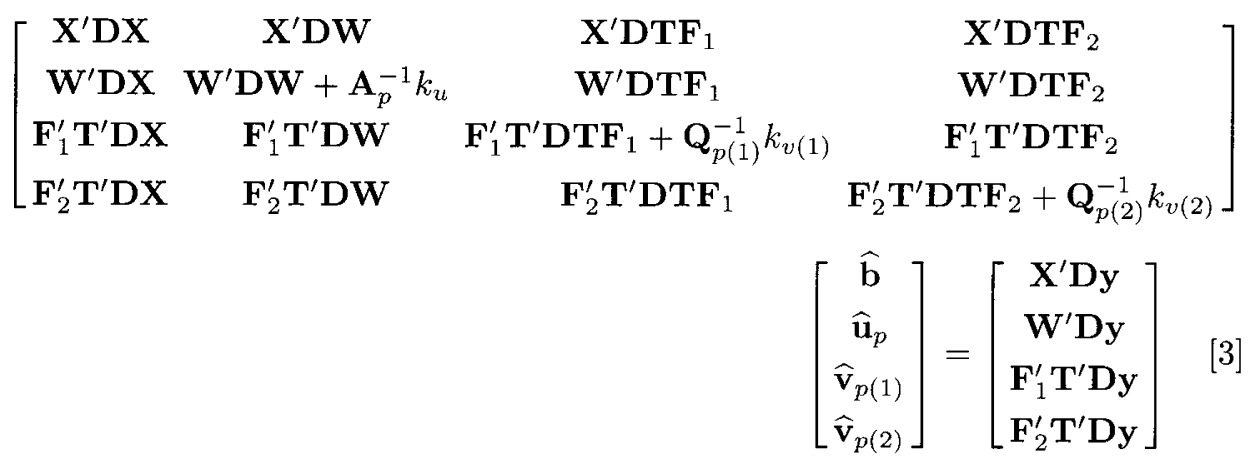


where $k_{u}=\sigma_{e}^{2} / \sigma_{u}^{2}, k_{v(i)}=\sigma_{e}^{2} / \sigma_{v(i)}^{2}$ and

$$
\begin{aligned}
\mathbf{D} & =\mathbf{D}_{1}-\mathbf{D}_{1}\left(\mathbf{D}_{1}+\Delta_{u}^{-1} k_{u}\right)^{-1} \mathbf{D}_{1} \\
\mathbf{D}_{1} & =\mathbf{D}_{2}-\mathbf{D}_{2} \mathbf{T}\left(\mathbf{T}^{\prime} \mathbf{D}_{2} \mathbf{T}+\Delta_{v(1)}^{-1} k_{v(1)}\right)^{-1} \mathbf{T}^{\prime} \mathbf{D}_{2} \\
\mathbf{D}_{2} & =\mathbf{R}^{-1}-\mathbf{R}^{-1} \mathbf{T}\left(\mathbf{T}^{\prime} \mathbf{R}^{-1} \mathbf{T}+\Delta_{v(2)}^{-1} k_{v(2)}\right)^{-1} \mathbf{T}^{\prime} \mathbf{R}^{-1}
\end{aligned}
$$

with the $\Delta$ matrices defined in equation [2]. Matrix $\mathbf{D}$, which results from successive absorption of the Mendelian sampling terms for the polygenic component and the QTLs, can be shown to be always diagonal and very simple to compute, even when several QTLs $(t>2)$ are fitted. Let $\delta_{v(i j k)}$ represent the Mendelian sampling term pertaining to $v$ effect $k(k=1,2)$ of individual $j$ at QTL $i$, and $\delta_{u(j)}$ the Mendelian sampling term for the polygenic effect of $j$. Then, the element of $\mathbf{D}$ pertaining to individual $j\left(d_{j j}\right)$ is computed as follows:

$$
d_{v}=r^{j j}
$$

For $i=t$ to $i=1$ compute

$$
\begin{aligned}
x & =\left(\delta_{v(i j 1)}+\delta_{v(i j 2)} /\left[\left(\delta_{v(i j 1)}+\delta_{v(i j 2)}\right) d_{v}+\delta_{v(i j 1)} \delta_{v(i j 2)} k_{v}\right]\right. \\
d_{v} & =d_{v}-d_{v}^{2} x
\end{aligned}
$$

and

$$
d_{j j}=d_{v}-d_{v}^{2}\left(1 / d_{v}+\delta_{u(j)} k_{u}\right)
$$

where $r^{j j}$ is the $j$ th diagonal element of $\mathbf{R}^{-1}$.

\section{REML analysis}

The REML analysis was performed using interval mapping and a derivative-free algorithm to maximize the likelihood for any given set of QTL positions, as described by Grignola et al (1996a) for a single QTL model. The log residual likelihood for the animal model was obtained by adding correction terms to the residual likelihood formed directly from the RAM MME (Grignola et al, 1996a). The RAM residual likelihood is:

$$
\begin{aligned}
L_{R A M} \propto & -0.5 \log \left|\mathbf{G}_{R A M}\right|-0.5\left(N-N F-N R_{R A M}\right) \log \left(\widehat{\sigma}_{e}^{2}\right) \\
& -0.5 \log \left|\mathbf{C}_{R A M}\right|
\end{aligned}
$$

where

$$
\widehat{\sigma}_{e}^{2}=\mathbf{y}^{\prime} \mathbf{P y} /(N-N F)
$$

where $N$ is the number of phenotypic observations, $N F$ the number of estimable fixed effects (rank of $\mathbf{X}$ ), $N R_{R A M}$ the number of random genetic effects of the parents $\left((1+2 t)\right.$ times the number of parents), $\mathbf{C}_{R A M}$ is the coefficient matrix in the left-hand-side of [3], $\mathbf{P}=\mathbf{V}^{-1}-\mathbf{V}^{-1} \mathbf{X}\left(\mathbf{X}^{\prime} \mathbf{V}^{-1} \mathbf{X}\right)^{-1} \mathbf{X}^{\prime} \mathbf{V}^{-1}, \mathbf{V}=\operatorname{Var}(\mathbf{y}) / \sigma_{e}^{2}$, and $\mathbf{G}_{R A M}$ is a block-diagonal with blocks $\mathbf{A}_{p} \sigma_{u}^{2}$ and $\mathbf{Q}_{p(i)} \sigma_{v(i)}^{2}$ for $i=1, \ldots, t$ (see also Meyer, 1989). 
The RAM residual likelihood is modified to obtain the residual likelihood for the animal model as follows (Grignola et al 1996a):

$$
L_{A M}=L_{R A M}-0.5 \log \left|\Delta^{-1}\right|-0.5 \log \left|\mathbf{C}_{22}\right|+0.5\left(N R-N R_{R A M}\right) \log \left(\widehat{\sigma}_{e}^{2}\right)
$$

where $\Delta$ is the block-diagonal with blocks $\Delta_{u}$ and $\Delta_{v(i)}(i=1, \ldots, t)$ from [2], $\mathbf{C}_{22}$ is the part of the MME for model [2] pertaining to $\mathbf{m}$ and $\varepsilon_{i}(i=1, \ldots, t)$, and $N R$ is total number of random genetic effects $[(1+2 t)$ times the number of animals] in the animal model.

The analysis is conducted in the form of interval mapping as in Grignola et al (1996a), except that now a $t$-dimensional search on a grid of combinations of positions of the $t$ QTLs must be performed. More precisely, we performed cyclic maximization by optimizing the position of the first QTL while holding the position of the second QTL constant and subsequently fixing the position of the first QTL while optimizing the position of the second QTL, etc. A minimum distance was allowed between the QTLs, which was determined such that the QTLs were always separated by two markers. Whittaker et al (1996) showed that for regression analysis and F2 or backcross designs, the two locations and effects of two QTLs in adjacent marker intervals are not jointly estimable. With other methods and designs, locations and variances of two QTLs in adjacent intervals should be either not estimable or poorly estimated. At each combination of $d_{1}$ and $d_{2}$ values, the residual likelihood is maximized with respect to the parameters $h^{2}, v_{i}^{2}(i=1, \ldots, t)$ and $\sigma_{e}^{2}$.

Matrices $\mathbf{Q}_{p(i)}, \mathbf{F}_{i}$ and $\Delta_{v(i)}$ were calculated for each QTL as described in Grignola et al (1996a).

\section{Hypothesis testing}

The presence of at least one QTL on the chromosome harboring the marker linkage group can be tested by maximizing the likelihood under the one-QTL model and under a polygenic model with no QTL fitted (Grignola et al, 1996a). The distribution of the likelihood ratio statistic for these two models can be obtained via simulation or data permutation (Churchill and Doerge, 1994; Grignola et al, 1996a, b; Uimari et al, 1996). Here, we consider testing the one-QTL model against the two-QTL model. This test is performed by comparing the maximized residual likelihood under the two-QTL model with (i) the maximized residual likelihood under the one-QTL model, (ii) the residual likelihood maximized under the oneQTL model with QTL position fixed at the REML estimate of $d_{1}$ obtained under the two-QTL model, and (iii) the residual likelihood maximized under the one-QTL model with QTL position fixed at the REML estimate of $d_{2}$ obtained under the two-QTL model. The distribution of these likelihood ratio statistics is not known, and obtaining it via data permutation would be difficult computationally, as many permutations would need to be analyzed, and as the two-dimensional search took 1-2 h of run-time for the design described below. The likelihood ratios corresponding to (i) $\left(\mathrm{LR}_{d}\right)$, (ii) $\left(\mathrm{LR}_{d 1}\right)$, and (iii) $\left(\mathrm{LR}_{d 2}\right)$ should have an asymptotic chi-square distribution within 1 and 3 degrees of freedom. When using $\mathrm{LR}_{d 1}$ and $\mathrm{LR}_{d 2}$, both ratios have to be significant in order to reject the null hypothesis of one QTL. This test is an intersection-union test (Casella and Berger, 1990; Berger, 1996), where 
for the first likelihood ratio the hypotheses are: $\mathrm{H}_{0}: \sigma_{v(1)}^{2} \neq 0$ and $\sigma_{v(2)}^{2}=0$ versus $\mathrm{H}_{1}: \sigma_{v(1)}^{2} \neq 0$ and $\sigma_{v(2)}^{2} \neq 0$, and for the second likelihood ratio the hypotheses are: $\mathrm{H}_{0}: \sigma_{v(1)}^{2}=0$ and $\sigma_{v(2)}^{2} \neq 0$ versus $\mathrm{H}_{1}: \sigma_{v(1)}^{2} \neq 0$ and $\sigma_{v(2)}^{2} \neq 0$. The intersectionunion test constructed in this way can be quite conservative, as its size may be much less than its specified value $\alpha$. For genome-wide testing, the significance level should also be adjusted for the number of independent tests performed (the number of chromosomes analyzed times the number of independent traits).

\section{SIMULATION}

\section{Design}

The design simulated was a granddaughter design (GDD) as in the single QTL study of Grignola et al (1996b), where marker genotypes are available on sons and phenotypes on daughters of the sons. The structure resembled the real GDD of the US public gene mapping project for dairy cattle based on the dairy bull DNA repository ( $\mathrm{Da}$ et al, 1994). The simulated GDD consisted of 2000 sons, 20 sires, and nine ancestors of the sires (fig 1).

Grandfather of Sire
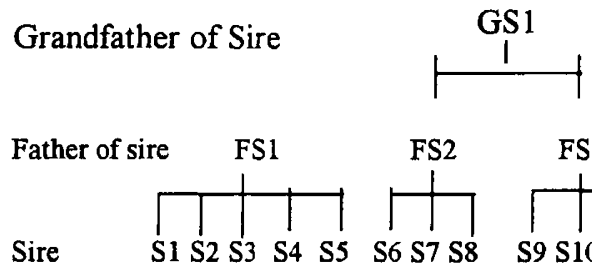
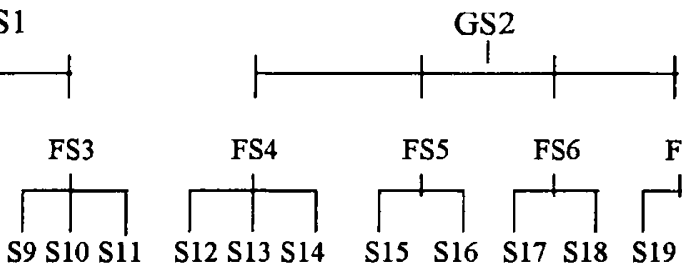

Fig 1. Pedigree of simulated granddaughter design.

The phenotype simulated was daughter yield deviation (DYD) of sons (VanRaden and Wiggans, 1991). DYD is an average of the phenotypes of the daughters adjusted for systematic environmental effects and genetic values of the daughters' dams. For details about the analysis of DYDs, see Grignola et al (1996b).

Marker and QTL genotypes were simulated according to Hardy-Weinberg frequencies and the map positions of all loci. All loci were in the same linkage group. Each marker locus had five alleles at equal frequencies. Several designs were considered which differed in the map positions of the two QTLs, in the number of markers, and in the proportion of the additive genetic variance explained by the two QTLs. These designs are defined in table II. Also simulated was a single QTL at $45 \mathrm{cM}$ to test the two-QTL analysis with data generated under the single QTL model.

Polygenic and QTL effects were simulated according to the pedigree in figure 1 . Data were analyzed by using the pedigree information on the sires. Note that in the simulation, no linkage disequilibrium (across families) was generated, ie, covariances between pairs of effects at different QTLs or between QTL and 
Table I. Definition of parameters and test statistics.

\begin{tabular}{ll}
\hline Parameter & \\
\hline$h^{2}$ & Narrow sense heritability \\
$v_{1}^{2}$ & Ratio of QTL 1 allelic to additive genetic variance \\
& QTL allelic variance equals half the additive variance due to the QTL) \\
$v_{2}^{2}$ & Ratio of QTL 2 allelic to additive genetic variance \\
$\sigma_{e}^{2}$ & Residual variance \\
$d_{1}$ & Map position of QTL 1 in Morgan (for two-QTL model) \\
$d_{2}$ & Map position of QTL 2 in Morgan (for two-QTL model) \\
$d$ & Map position of QTL in Morgan (for one-QTL model) \\
$\mathrm{LR}_{d 1}$ & Likelihood ratio statistic from the residual likelihood maximized \\
& under the two-QTL model and the residual likelihood maximized \\
& under the one-QTL model with QTL position fixed at the REML \\
& estimate of $d_{1}$ from the two-QTL model \\
$\mathrm{LR}_{d 2}$ & Likelihood ratio statistic from the residual likelihood maximized \\
& under, the two-QTL model and the residual likelihood maximized \\
& under the one-QTL model with QTL position fixed at the REML \\
estimate of $d_{2}$ from the two-QTL model & Likelihood ratio statistic from the likelihood maximized under \\
the two-QTL model and the likelihood maximized under \\
the one-QTL model \\
$\mathrm{LR}_{d}$
\end{tabular}

polygenic effects were zero. Therefore, an additional design was simulated where linkage disequilibrium was generated by simulating DYDs also for sires, creating a larger number of sires and culling those sires with DYD lower than the 90th percentile of the DYD distribution. QTL positions for this design were $30 \mathrm{cM}$ (interval 2) and $70 \mathrm{cM}$ (interval 3) with five markers, and the QTL model was the normal-effects model (see below). Estimates of the simulated correlations (SE in parentheses), across 30 replicates, were $-0.20(0.05),-0.33(0.04)$, and -0.32 (0.04), between pairs of $v$ effects at QTL 1 and QTL 2, between pairs of $v$ effects at QTL 1 and polygenic effects, and between pairs of $v$ effects at QTL 2 and polygenic effects, respectively. The effects of one or several generations of phenotypic truncation selection on additive genetic variance in a finite locus model has been studied analytically by Hospital and Chevalet (1996). 


\section{QTL models}

Two different QTL models were used to simulate data. Under both models, phenotypes were simulated as

$$
\mathrm{DYD}_{j}=\frac{1}{n_{j}} \sum_{i=1}^{2} \sum_{k=1}^{n_{j}} g_{i j k}+\mathbf{u}_{j}+\mathbf{e}_{j} ; \quad \operatorname{Var}\left(e_{j}\right)=\frac{1}{n_{j}}\left(0.75 \sigma_{u}^{2}+\sigma_{e}^{2}\right)
$$

where $n_{j}$ was the number of daughters of son $j, g_{i j k}$ was the sum of the $v$ effects in daughter $k$ of son $j$ at QTL $i, \mathbf{u}_{j}$ was a normally distributed polygenic effect, $\mathbf{e}_{j}$ was a normally distributed residual, polygenic variance $\left(\sigma_{u}^{2}\right)$ was equal to the difference between additive genetic variance $\left(\sigma_{a}^{2}\right)$ and the variance explained by the QTLs, and $\sigma_{e}^{2}$ was environmental variance. Number of daughters per son was set to 50 , corresponding to a reliability (Van Raden and Wiggans, 1991) near 0.8. Narrow sense heritability of individual phenotypes was $h^{2}=0.3$, and phenotypic SD was $\sigma_{p}=100$.

Note that the QTL contribution to the DYDs of sons was generated by sampling individual QTL allelic effects of daughters under each of the two genetic models described below. This sampling of QTL effects ensures that DYD of a heterozygous son, or of a son with substantial difference in the additive effects of the alleles at a QTL, has larger variance among daughters due to the QTL than a homozygous son or a son with similar QTL allelic effects.

Two different models were used to describe variation at the QTL, which are identical to two of the models considered by Grignola et al (1996b).

\section{Normal-effects model}

For each individual with both or one parent(s) unknown, both or one effect(s) at QTL $k(k=1,2)$ were drawn from $\mathrm{N}\left(0, \sigma_{v(k)}^{2}\right)$. For the pedigree in figure 1 , there were 32 base alleles, and each QTL was treated as a locus with 32 distinct alleles in passing on alleles to descendants. The parameter $\sigma_{v(k)}^{2}$ was set to $0.125 \sigma_{a}^{2}$ or $0.625 \sigma_{a}^{2}$, ie, QTL $k$ accounted for $25 \%\left(2 v_{k}^{2}=0.25\right)$ or $12.5 \%\left(2 v_{k}^{2}=0.125\right)$ of the total additive genetic variance, respectively.

Consequently, the two QTLs accounted jointly for between 25 and $50 \%$ of the additive genetic variance.

\section{Biallelic model}

Each QTL was biallelic with allele frequency $p_{1}=p_{2}=p=0.5$. The variance at QTL $k$ was

$$
2 \sigma_{v(k)}^{2}=2 v_{k}^{2} \sigma_{a}^{2}=2 p(1-p) a_{k}^{2}
$$

where for $p=0.5$ and $2 v_{k}^{2}=0.25$ or $2 v_{k}^{2}=0.125$, half the homozygote difference at QTL $k, a_{k}$, and allelic variance $\sigma_{v(k)}^{2}$ were determined. 


\section{RESULTS}

The designs studied are described in table II and differ in the QTL positions, in the number of markers, and in the proportion of the additive genetic variance explained jointly by two linked QTLs. Overall, the QTL parameters were estimated quite accurately as in the single-QTL analysis of Grignola et al (1996b), except that there was a tendency to overestimate the distance between the QTLs with decreasing true distance.

Table II. Simulated designs (IA, $\ldots$, IVB) differing in the QTL positions $\left(d_{1}, d_{2}\right)$, number of (\#) markers, and percentage of the additive genetic variance $\left(\sigma_{a}^{2}\right)$ explained jointly by the QTLs.

\begin{tabular}{lllllllll}
\hline & $I A$ & $I B$ & $I I A$ & $I I B$ & $I I I A$ & $I I I B$ & $I V A$ & IVB \\
\hline$d_{1}, d_{2}(\mathrm{cM})$ & 30,70 & 30,70 & 25,55 & 25,55 & 25,45 & 25,45 & 25,45 & 25,45 \\
$\#$ markers & 5 & 5 & 9 & 9 & 9 & 9 & 5 & 5 \\
$\% \sigma_{a}^{2}$ & 50 & 25 & 50 & 25 & 50 & 25 & 50 & 25 \\
\hline
\end{tabular}

Parameter estimates for all designs in table II and for the normal-effects QTL model used in the data simulations are presented in table III. There appeared to be a slight tendency to overestimate the QTL variance contributions $\left(v^{2}\right)$, but, in most cases not significantly. The QTL map positions and the distance between the QTLs were estimated accurately when the true map distance between the QTLs was 30 or $40 \mathrm{cM}$. When the true map distance was only $20 \mathrm{cM}$, there was a tendency to overestimate the QTL distance. This overestimation was significantly more pronounced when the number of markers was reduced from nine (every $10 \mathrm{cM}$, designs IIIA, B) to five (every $20 \mathrm{cM}$, designs IVA, B). To investigate whether the overestimation of the QTL distance was related to the search strategy requiring a minimum distance between the QTLs such that these were always separated by two markers (with the exception of designs IVA, B), the minimum distance was reduced to 10 and $2 \mathrm{cM}$. However, parameter estimates and likelihood ratios remained unchanged.

When the QTLs accounted jointly for only $25 \%$ of the additive genetic variance as compared to $50 \%$, there was little change in the precision of the estimates of the QTL variance contributions. Standard errors of the QTL positions were higher, and overestimation of the distance between QTLs only $20 \mathrm{cM}$ apart was slightly more pronounced.

Parameter estimates for designs simulated under the biallelic QTL model are shown in table V. Except for the QTL model, these designs are identical to designs IA, B and IIIA, B in table II. Parameters were estimated with an accuracy not noticeably lower than for the normal-effects QTL model, an observation in agreement with the single-QTL study of Grignola et al (1996b).

When analyzing the designs in table II with the single-QTL model, the most likely QTL position ( $d$ in tables III and V) was always somewhere in between the QTL positions estimated under the two-QTL model. Averaged across replicates, 
Table III. Parameter estimates (SE in parentheses) for designs ${ }^{\mathrm{a}}$ with the normal-effects QTL model (30 replicates per design).

\begin{tabular}{lcccc}
\hline Parameter $^{\mathrm{b}}$ & IA & IIA & IIIA & IVA \\
& $I B$ & $I I B$ & IIIB & IVB \\
\hline$h^{2}$ & $0.553(0.050)$ & $0.570(0.046)$ & $0.478(0.054)$ & $0.483(0.045)$ \\
& $0.495(0.047)$ & $0.521(0.047)$ & $0.470(0.048)$ & $0.470(0.049)$ \\
$v_{1}^{2}$ & $0.130(0.010)$ & $0.139(0.012)$ & $0.107(0.010)$ & $0.142(0.014)$ \\
& $0.065(0.006)$ & $0.072(0.011)$ & $0.091(0.014)$ & $0.090(0.011)$ \\
$v_{2}^{2}$ & $0.135(0.012)$ & $0.135(0.013)$ & $0.137(0.014)$ & $0.133(0.015)$ \\
& $0.065(0.007)$ & $0.068(0.009)$ & $0.068(0.007)$ & $0.066(0.011)$ \\
$\sigma_{e}^{2}$ & $767.2(102.3)$ & $888.7(96.8)$ & $740.9(102.8)$ & $921.3(96.2)$ \\
& $887.0(95.9)$ & $869.2(103.0)$ & $989.5(116.8)$ & $968.4(109.8)$ \\
$d_{1}$ & $0.282(0.009)$ & $0.252(0.008)$ & $0.231(0.010)$ & $0.219(0.011)$ \\
& $0.298(0.018)$ & $0.246(0.015)$ & $0.224(0.017)$ & $0.228(0.018)$ \\
$d_{2}$ & $0.722(0.006)$ & $0.560(0.006)$ & $0.462(0.010)$ & $0.506(0.018)$ \\
& $0.692(0.011)$ & $0.568(0.014)$ & $0.485(0.020)$ & $0.515(0.024)$ \\
$d$ & $0.493(0.029)$ & $0.420(0.022)$ & $0.362(0.011)$ & $0.336(0.011)$ \\
& $0.501(0.032)$ & $0.336(0.023)$ & $0.345(0.013)$ & $0.349(0.016)$ \\
\hline
\end{tabular}

${ }^{\mathrm{a}}$ Designs are defined in table II; ${ }^{\mathrm{b}}$ parameters are defined in table I.

the estimated QTL position was very near the mean of the true positions. This result was expected, as both QTLs had equal variance contributions and on average equally informative flanking markers.

Likelihood ratio statistics for all designs in table II and for the normal-effects QTL model are presented in table IV. The average values of the likelihood ratio statistics for testing between the single- and two-QTL models declined as expected with decreasing distance between the two QTLs (designs IIA, B versus designs IIIA, $\mathrm{B}$ ), with decreasing number of markers (designs IA, B versus IIA, B, and designs IIIA, B versus designs IVA, B), and with decreasing joint variance contribution of the QTLs (A versus B). The average value of the likelihood ratio statistic $\mathrm{LR}_{d}$ was always considerably lower than those of $\mathrm{LR}_{d 1}$ and $\mathrm{LR}_{d 2}$.

The power figures in table IV were calculated assuming that $\mathrm{LR}_{d 1}, \mathrm{LR}_{d 2}$ and $\mathrm{LR}_{d}$ follow either a chi-square distribution with $1 \mathrm{df}$ or $3 \mathrm{df}$ and using an $\alpha$ value of $0.05 / 29=0.0017$. To allow for any interpretation of these power figures, we estimated the type-I error by simulating data with a single QTL explaining either $25,12.5$ or $6.25 \%$ of the additive genetic variance. For the type-I error estimation, $\alpha$ was set to 0.05 , and the number of replicates was 200. Estimates of type-I errors are in table VI, for the two tests $\left(\mathrm{LR}_{d}\right.$ and $\mathrm{LR}_{d 1}$ and $\left.\mathrm{LR}_{d 2}\right)$ and for thresholds from chi-square distributions with 1, 2 and 3 df. Type-I errors tended to increase slightly with size of the QTL variance, and were consistently lower for the test using LR $_{d}$. Based on these results, the empirical type-I error was close to the pre-specified value of 0.05 for the $L_{d 1}$ and $L R_{d 2}$ tests when using the $\chi_{1}^{2}$-threshold, while it was consistently too low for the $\mathrm{LR}_{d}$ test. 
Table IV. Likelihood ratio statistics (SE in parentheses) and powers of detecting a second QTL for designs ${ }^{\mathrm{a}}$ with the normal-effects QTL model (30 replicates per design).

\begin{tabular}{|c|c|c|c|c|c|c|c|c|}
\hline Statistic/Power ${ }^{\mathbf{b}}$ & \multicolumn{2}{|c|}{$\begin{array}{l}I A \\
I B \\
\end{array}$} & \multicolumn{2}{|c|}{$\begin{array}{l}I I A \\
I I B\end{array}$} & \multicolumn{2}{|c|}{$\begin{array}{l}I I I A \\
I I I B \\
\end{array}$} & \multicolumn{2}{|c|}{$\begin{array}{l}I V A \\
I V B\end{array}$} \\
\hline $\mathrm{LR}_{d 1}$ & \multicolumn{2}{|c|}{$\begin{array}{l}40.102(3.6) \\
15.708(2.4)\end{array}$} & \multicolumn{2}{|c|}{$\begin{array}{l}51.378(4.9) \\
27.594(3.2)\end{array}$} & \multicolumn{2}{|c|}{$\begin{array}{l}34.967(4.1) \\
18.990(3.1)\end{array}$} & \multicolumn{2}{|c|}{$\begin{array}{l}25.974(3.4) \\
18.825(3.2)\end{array}$} \\
\hline $\mathrm{LR}_{d 2}$ & \multicolumn{2}{|c|}{$\begin{array}{l}40.626(3.2) \\
14.270(1.7)\end{array}$} & \multicolumn{2}{|c|}{$\begin{array}{l}49.930(6.1) \\
18.053(2.6)\end{array}$} & \multicolumn{2}{|c|}{$\begin{array}{l}27.647(3.9) \\
14.644(2.0)\end{array}$} & \multicolumn{2}{|c|}{$\begin{array}{l}24.017(3.7) \\
11.774(2.1)\end{array}$} \\
\hline $\mathrm{LR}_{d}$ & \multicolumn{2}{|c|}{$\begin{array}{r}23.901(2.3) \\
6.767(0.9)\end{array}$} & \multicolumn{2}{|c|}{$\begin{array}{l}29.232(3.1) \\
10.893(1.3)\end{array}$} & \multicolumn{2}{|c|}{$\begin{array}{r}10.860(1.2) \\
4.568(0.7)\end{array}$} & \multicolumn{2}{|c|}{$\begin{array}{l}7.354(0.9) \\
3.495(0.7)\end{array}$} \\
\hline $\mathrm{PWR}_{d 1, d 2}{ }^{\mathrm{c}}$ & $\begin{array}{l}0.97 \\
0.50\end{array}$ & $\begin{array}{l}0.90 \\
0.27\end{array}$ & $\begin{array}{l}0.83 \\
0.77\end{array}$ & $\begin{array}{l}0.73 \\
0.57\end{array}$ & $\begin{array}{l}0.83 \\
0.40\end{array}$ & $\begin{array}{l}0.73 \\
0.23\end{array}$ & $\begin{array}{l}0.77 \\
0.31\end{array}$ & $\begin{array}{l}0.47 \\
0.10\end{array}$ \\
\hline $\mathrm{PWR}_{d}$ & $\begin{array}{l}0.97 \\
0.40\end{array}$ & $\begin{array}{l}0.80 \\
0.27\end{array}$ & $\begin{array}{l}0.83 \\
0.73\end{array}$ & $\begin{array}{l}0.70 \\
0.47\end{array}$ & $\begin{array}{l}0.67 \\
0.27\end{array}$ & $\begin{array}{l}0.50 \\
0.03\end{array}$ & $\begin{array}{l}0.47 \\
0.14\end{array}$ & $\begin{array}{l}0.17 \\
0.03\end{array}$ \\
\hline
\end{tabular}

${ }^{a}$ Designs are defined in table II; ${ }^{b}$ likelihood ratios are defined in table I. ${ }^{c}$ first number using $\alpha=0.0017$ and the $1 \mathrm{df}$ chi-square distribution; second number using the $3 \mathrm{df}$ chisquare distribution.

With this background, the power of rejecting the single-QTL model based on requiring both $\mathrm{LR}_{d 1}$ and $\mathrm{LR}_{d 2}$ to exceed the significance threshold was as expected always higher than or equal to the power of the $\mathrm{LR}_{d}$ statistic. For the test based on $\mathrm{LR}_{d 1}$ and $\mathrm{LR}_{d 2}$, power declined as expected with decreasing distance between QTLs and with decreasing true QTL variance contribution. For the joint QTL variance contribution of $50 \%$ and the test based on $\mathrm{LR}_{d 1}$ and $\mathrm{LR}_{d 2}$, power was equal to or higher than 0.5 always for the $\chi_{1}^{2}$ threshold and always except for design IVA for the $\chi_{3}^{2}$ threshold. For the joint QTL variance contribution of $25 \%$, a power of at least 0.5 was achieved only for the $30 \mathrm{cM}$ distance between QTLs and the $\chi_{1}^{2}$ and $\chi_{3}^{2}$ thresholds. This finding must be interpreted by keeping in mind the choice of $\alpha=0.0017$ and the fact that the test, as contructed here, is rather conservative.

For the data simulated with a single QTL explaining either 25 or $12.5 \%$ of the additive genetic variance, the map positions estimated under the two-QTL model were near the true position and a ghost position to either side of the true position in most replicates. This behavior of the REML analysis seems to support the use of $\mathrm{LR}_{d 1}$ and $\mathrm{LR}_{d 2}$ instead of $\mathrm{LR}_{d}$.

Likelihood ratio statistics for the biallelic QTL model and some of the designs in table II are presented in table V. Overall, likelihood ratios and power figures were similar to those for the normal-effects QTL model, with somewhat lower power for design IA but slightly higher power for other designs. These differences are most likely due to the limited number of replications (30).

The cyclic maximization strategy for the two-QTL model took about $20 \mathrm{~min}$ of serial wall-clock time on a 21-processor IBM SP2 system for a chromosome of $80 \mathrm{cM}$ length, compared with $1.5 \mathrm{~h}$ for a two-dimensional search. Run-time for the single-QTL analysis was at most $8 \mathrm{~min}$.

For the designs in table II, the two QTLs had equal variance contributions. Therefore, additional designs with QTL positions of 30 and $70 \mathrm{cM}$ (five markers) 
Table V. Parameter estimates (SE in parentheses) and likelihood ratio statistics for designs $^{\mathrm{a}}$ with the biallelic QTL model (30 replicates per design).

\begin{tabular}{|c|c|c|c|c|c|c|c|c|}
\hline Parameter ${ }^{\mathrm{b}}$ & \multicolumn{2}{|c|}{$I A$} & \multicolumn{2}{|c|}{$I B$} & \multicolumn{2}{|c|}{ IIIA } & \multicolumn{2}{|c|}{$I I I B$} \\
\hline$h^{2}$ & \multicolumn{2}{|c|}{$0.536(0.045)$} & \multicolumn{2}{|c|}{$0.552(0.051)$} & \multicolumn{2}{|c|}{$0.501(0.034)$} & \multicolumn{2}{|c|}{$0.516(0.049)$} \\
\hline$v_{1}^{2}$ & \multicolumn{2}{|c|}{$0.144(0.014)$} & \multicolumn{2}{|c|}{$0.065(0.006)$} & \multicolumn{2}{|c|}{$0.117(0.011)$} & \multicolumn{2}{|c|}{$0.074(0.009)$} \\
\hline$v_{2}^{2}$ & \multicolumn{2}{|c|}{$0.132(0.011)$} & \multicolumn{2}{|c|}{$0.073(0.008)$} & \multicolumn{2}{|c|}{$0.147(0.012)$} & \multicolumn{2}{|c|}{$0.074(0.008)$} \\
\hline$\sigma_{e}^{2}$ & \multicolumn{2}{|c|}{$783.7(87.7)$} & \multicolumn{2}{|c|}{$799.8(111.8)$} & \multicolumn{2}{|c|}{$765.8(78.1)$} & \multicolumn{2}{|c|}{$853.6(102.9)$} \\
\hline$d_{1}$ & \multicolumn{2}{|c|}{$0.280(0.014)$} & \multicolumn{2}{|c|}{$0.264(0.011)$} & \multicolumn{2}{|c|}{$0.220(0.011)$} & \multicolumn{2}{|c|}{$0.202(0.015)$} \\
\hline$d_{2}$ & \multicolumn{2}{|c|}{$0.706(0.012)$} & \multicolumn{2}{|c|}{$0.716(0.009)$} & \multicolumn{2}{|c|}{$0.459(0.008)$} & \multicolumn{2}{|c|}{$0.464(0.012)$} \\
\hline$d$ & \multicolumn{2}{|c|}{$0.511(0.028)$} & \multicolumn{2}{|c|}{$0.492(0.034)$} & \multicolumn{2}{|c|}{$0.362(0.011)$} & \multicolumn{2}{|c|}{$0.341(0.015)$} \\
\hline $\mathrm{LR}_{d 1}$ & \multicolumn{2}{|c|}{$42.828(3.8)$} & \multicolumn{2}{|c|}{$16.622(1.9)$} & \multicolumn{2}{|c|}{$29.563(3.6)$} & \multicolumn{2}{|c|}{$15.670(2.3)$} \\
\hline $\mathrm{LR}_{d 2}$ & \multicolumn{2}{|c|}{$45.062(4.8)$} & \multicolumn{2}{|c|}{$19.792(2.3)$} & 43.2 & $(5.4)$ & 18.4 & (2.6) \\
\hline $\mathrm{LR}_{d}$ & 22.7 & (2.9) & 9.7 & (1.2) & 14.7 & (1.6) & $5.7 \mathrm{~s}$ & $(0.7)$ \\
\hline $\mathrm{PWR}_{d 1, d 2}{ }^{\mathrm{c}}$ & 0.90 & 0.83 & 0.72 & 0.34 & 0.87 & 0.77 & 0.53 & 0.27 \\
\hline $\mathrm{PWR}_{d}$ & 0.87 & 0.67 & 0.62 & 0.24 & 0.83 & 0.57 & 0.40 & 0.07 \\
\hline
\end{tabular}

${ }^{a}$ Designs are defined in table II; ${ }^{b}$ parameters and likelihood ratios are defined in table I;

${ }^{c}$ first number using $\alpha=0.0017$ and the $1 \mathrm{df}$ chi-square distribution; second number using the $3 \mathrm{df}$ chi-square distribution.

Table VI. Estimated type-I errors based on 200 replicates for two tests $\left(\mathrm{LR}_{d}\right.$ and $\mathrm{LR}_{d 1}$ and $\mathrm{LR}_{d 2}$ ) defined in the text and for three designs based on the normal-effects QTL model and different ratios of QTL to additive genetic variance $\left(v^{2}\right)$, using thresholds from chi-square distributions with 1,2 or $3 \mathrm{df}$.

\begin{tabular}{lcccc}
\hline & $\mathrm{v}^{2}$ & $\chi_{1}^{2}$ & $\chi_{2}^{2}$ & $\chi_{3}^{2}$ \\
\cline { 3 - 5 } $\mathrm{LR}_{d}$ & 0.0625 & $1 \%$ & $0 \%$ & $0 \%$ \\
$\mathrm{LR}_{d 1}$ and $\mathrm{LR}_{d 2}$ & & $5 \%$ & $1 \%$ & $0 \%$ \\
$\mathrm{LR}_{d}$ & 0.125 & $1 \%$ & $0 \%$ & $0 \%$ \\
$\mathrm{LR}_{d 1}$ and $\mathrm{LR}_{d 2}$ & & $6 \%$ & $2 \%$ & $0 \%$ \\
$\mathrm{LR}_{d}$ & 0.25 & $2 \%$ & $1 \%$ & $0 \%$ \\
$\mathrm{LR}_{d 1}$ and $\mathrm{LR}_{d 2}$ & & $7 \%$ & $2 \%$ & $1 \%$ \\
\hline
\end{tabular}

and 25 and $45 \mathrm{cM}$ (nine markers), respectively, were simulated using the normaleffect QTL model, with QTL 1 explaining 25\% and QTL $212.5 \%$ of the additive genetic variance. The average estimates (with $\mathrm{SE}$ in parentheses) of QTL position from the single QTL analysis were $0.396 \mathrm{M}(0.023)$ and $0.298 \mathrm{M}(0.010)$ for the 30 and $70 \mathrm{cM}$ and 25 and $45 \mathrm{cM}$ designs, respectively, being closer to the first locus with the larger variance contribution. Estimated QTL positions from the two-QTL analysis were $0.285 \mathrm{M}(0.008)$ and $0.720 \mathrm{M}(0.010)$ for the 30 and $70 \mathrm{cM}$ design, and $0.242 \mathrm{M}(0.020)$ and $0.440 \mathrm{M}(0.019)$ for the 25 and $45 \mathrm{cM}$ design. Average $v^{2}$ estimates were $0.143(0.013)$ and $0.072(0.010)$ for the first design, and $0.126(0.019)$ 
and $0.077(0.012)$ for the second design, respectively. For the 30 and $70 \mathrm{cM}$ design, power was $0.47(0.40)$ for the $\chi_{1}^{2}\left(\chi_{3}^{2}\right)$ threshold and the test based on $\mathrm{LR}_{d 1}$ and $\mathrm{LR}_{d 2}$. For the 25 and $45 \mathrm{cM}$ design, power was only $0.33(0.10)$ for the same tests.

When linkage disequilibrium was generated by phenotypic truncation selection of sires for the design with QTL positions of 30 and $70 \mathrm{cM}$ and joint QTL genetic variance contribution of $50 \%$, QTL parameters and their estimates were clearly affected. Heritability was estimated low $(0.213 \pm 0.020)$, and the $v_{k}^{2}(k=1,2)$ were estimated high $(0.184 \pm 0.014,0.211 \pm 0.014)$, but QTL positions were estimated accurately $(0.292 \pm 0.010,0.716 \pm 0.007)$. Power appeared to be somewhat reduced compared to the same design without selection, and was estimated at 0.86 and 0.73 for the $\chi_{1}^{2}$ and $\chi_{3}^{2}$ thresholds, respectively. Reduction in power was probably due to the high estimate of error variance $(1737.9 \pm 55.3)$.

\section{CONCLUSIONS}

The REML analysis of Grignola et al (1996a, b), based on a mixed linear model with random and normally distributed QTL allelic effects and conditional on incomplete information from multiple linked markers, has been extended here to fit multiple linked QTLs. This extension is necessary to eliminate biases in the estimates of the QTL parameters position and variance, which occur when fitting a single QTL and other linked QTLs are present. For the present study, the analysis had been implemented for two QTLs on the same chromosome using a two-dimensional search. When fitting more than two linked QTLs or additional unlinked QTLs, a more efficient search strategy may be required, or alternative algorithms (eg, Meyer and Smith, 1996). In the meantime, a cyclic optimization approach was implemented, with one QTL position held constant while optimizing the other, and vice versa, which reduced the number of likelihood evaluations and hence CPU time considerably relative to a two-dimensional search. As likelihood maximizations at different position combinations are independent of each other, use of multiple processors, if available, would reduce run-times substantially.

For the one-QTL model, relationships between the REML analysis, the equivalent method of Xu and Atchley (1995), the method of Schork (1993), and the Bayesian analysis of Uimari et al (1996) were discussed in Grignola et al (1996a). The method of $\mathrm{Xu}$ and Atchley (1995) fitting variances associated with the nextto-flanking markers to account for additional linked QTLs would not have worked well for the designs studied here. A first reason is the inclusion of ancestors of the sires in the analysis, as their method fits random effects associated with the marker alleles in founders which erode across generations due to recombination. Another reason is the small number of families differing in the flanking and next-to-flanking markers, resulting in too little information for estimation of variances associated with the next-to-flanking markers in the method of $\mathrm{Xu}$ and Atchley (1995). For similar reasons, composite interval mapping (Zeng, 1994) and multiple QTL mapping (Jansen, 1993), which are based on the inclusion of markers as cofactors, are not suitable for the analysis of multi-generational pedigrees.

REML analysis under the two-QTL model yielded fairly accurate parameter estimates. Map distance between the QTLs was overestimated, with decreasing distance between the two QTLs and wider spacing of markers. As in the single QTL 
study, the REML analysis was robust to the number of alleles at the QTLs, as there was relatively little difference in parameter estimates and likelihood ratio statistics between designs generated with the normal-effects and biallelic QTL models, given the number of replicates performed. Previous linkage analyses (eg, Knott and Haley, 1992; Haley et al, 1994) lead to the conclusion that a minimum distance of $20 \mathrm{cM}$ was required between linked QTLs for their separate detection. This result was confirmed in the present study. However, discrimination among different numbers of QTL (eg, one versus two) requires additional research, including an investigation of alternative approaches such as an adaptation of composite interval mapping to pedigree analysis. Gains in power from fitting additional unlinked QTL and selection of QTL to be included in the analysis are related topics warranting further research.

If there is linkage disequilibirum due to selection, QTL positions will still be estimated accurately, while variance estimates and power may be affected. Accounting for disequilibrium in the analysis should be investigated.

\section{ACKNOWLEDGMENTS}

This research was supported by Award No 92-01732 of the National Research Initiative Competitive Grants Program of the US Department of Agriculture, by the Holstein Association USA, and by ABS Global Inc.

\section{REFERENCES}

Berger RL (1996) Likelihood ratio tests and intersection-union tests. In: Advances in Statistical Decision Theory (N Balakreshnan and S Panchapahesan, eds), Birkhauser, Boston (in press)

Bulmer MG (1985) The Mathematical Theory of Quantitative Genetics. Clarendon Press, Oxford

Cantet RJC, Smith C (1991) Reduced animal model for marker assisted selection using best linear unbiased prediction. Genet Sel Evol 23, 221-233

Casella G, Berger RL (1990) Statistical Inference. Wadsworth \& Brooks/Cole, Advanced Books \& Software, Pacific Grove, CA

Churchill G, Doerge R (1994) Empirical threshold values for quantitative trait mapping. Genetics 138, 963-971

Da Y, Ron M, Yanai A, Band M, Everts RE, Heyen DW, Weller JI, Wiggans GR, Lewin HA (1994) The Dairy Bull DNA Repository: A resource for mapping quantitative trait loci. Proc 5th World Congr Genetics Appl Livest Prod 21, 229-232

Fernando RL, Grossman M (1989) Marker-assisted selection using best linear unbiased prediction. Genet Sel Evol 21, 467-477

Goddard M (1992) A mixed model for analyses of data on multiple genetic markers. Theor Appl Genet 83, 878-886

Gotz KU, Ollivier L (1994) Theoretical aspects of applying sib-pair linkage tests to livestock species. Genet Sel Evol 24, 29-42

Grignola FE, Hoeschele I, Tier B (1996a) Mapping quantitative trait loci via residual maximum likelihood: I. Methodology. Genet Sel Evol 28, 479-490

Grignola FE, Hoeschele I, Zhang Q, Thaller G (1996b) Mapping quantitative trait loci via residual maximum likelihood: I. A simulation study. Genet Sel Evol 28, 491-504

Haley CS, Knott SA, Elsen J-M (1994) Mapping quantitative trait loci in crosses between outbred lines using least-squares. Genetics 136, 1195-1207 
Haseman JK, Elston RC (1972) The investigation of linkage between a quantitative trait and a marker locus. Behav Genet 2, 3-19

Hoeschele I (1993) Elimination of quantitative trait loci equations in an animal model incorporating genetic marker data. J Dairy Sci 76, 1693-1713

Hoeschele I, Uimari P, Grignola FE, Zhang Q, Gage KM (1996) Statistical mapping of polygene loci in livestock. Proc Int Biometrics Soc (in press)

Hospital F, Chevalet C (1996) Interactions of selection, linkage and drift in the dynamics of polygenic characters. Genet Res Camb 67, 77-87

Jansen RC (1993) Interval mapping of multiple quantitative trait loci. Genetics 135, $252-324$

Knott SA, Haley CS (1992) Maximum likelihood mapping of quantitative trait loci using full-sib families. Genetics 132, 1211-1222

Lander ES, Botstein D (1989) Mapping Mendelian factors underlying quantitative traits using RFLP linkage maps. Genetics 121, 185-199

Meyer K (1989) Restricted Maximum Likelihood to estimate variance components for animal models with several random effects using a derivative-free algorithm. Genet Sel Evol 21, 317-340

Meyer K, Smith SP (1996) Restricted Maximum Likelihood estimation for animal models using derivatives. Genet Sel Evol 28, 23-50

Schork NJ (1993) Extended multi-point identity-by-descent analysis of human quantitative traits: Efficiency, power, and modeling considerations. Am J Hum Genet 53, 1306-1319

Thaller G, Hoeschele I (1996a) A Monte Carlo method for Bayesian analysis of linkage between single markers and quantitative trait loci: I. Methodology. Theor Appl Genet 93, 1161-1166

Thaller G, Hoeschele I (1996b) A Monte Carlo method for Bayesian analysis of linkage between single markers and quantitative trait loci: I. A simulation study. Theor Appl Genet 93, 1167-1174

Uimari P, Thaller G, Hoeschele I (1996) The use of multiple linked markers in a Bayesian method for mapping quantitative trait loci. Genetics 143, 1831-1842

Uimari P, Hoeschele I (1996) Mapping linked quantitative trait loci with Bayesian analysis and Markov chain Monte Carlo algorithms. Genetics 146, 735-743

VanRaden PM, Wiggans GR (1991) Derivation, calculation, and use of national animal model information. J Dairy Sci 74, 2737-2746

Wang T, Fernando RL, van der Beek S, Grossman M (1995) Covariance between relatives for a marked quantitative trait locus. Genet Sel Evol 27, 251-274

Weller JI (1986) Maximum likelihood techniques for the mapping and analysis of quantitative trait loci with the aid of genetic markers. Biometrics 42, 627-640

Weller JI, Kashi Y, Soller M (1990) Power of daughter and granddaughter designs for determining linkage between marker loci and quantitative trait loci in dairy cattle. J Dairy Sci 73, 2525-2537

Whittaker JC, Thompson R, Visscher PM (1996) On the mapping of QTL by regression of phenotype on marker-type. Heredity 77, 23-32

Xu S, Atchley WR (1995) A random model approach to interval mapping of Quantitative Trait Loci. Genetics 141, 1189-1197

Zeng Z-B (1994) Precision mapping of quantitative trait loci. Genetics 136, 1457-1468 